\title{
Drop-size analysis using 2D Double Density Dual Tree Discrete Wavelet Transform
}

\author{
S. Tawade ${ }^{1}$, P. Ghonge ${ }^{2}$, K. Tuckley ${ }^{3}$ \\ ${ }^{1}$ Computer Science student, YadavraoTasgoankar Institute of Engineering and Technology, Karjat, Mumbai. \\ ${ }^{2}$ Ph.D student, RAIT, Nerul, New Mumbai, India; Senior Professor, RAIT, Nerul, New Mumbai, India \\ \{swati.tawade08@gmail.com; ghongepa@yahoo.com \}
}

\begin{abstract}
Thispaper proposes a method for raindrop measurement based on image processing using 2D double density dual tree DWT.Concept which make this project is to use a technique apart from disdrometer to abstract parameter values such as drop size from image.Trasform technique 2D double density dual tree DWT which is also know as framelet transform is four time as expansive compared to simple discrete signal.In Propsed method images are captured through experimental setup using two cameras with advanced features, in such a way that both captured images have same central point. Captured images are decomposed into two low frequency components and sixteen high frequency components using 2D double density dual tree transform. These components are fused together employing maximum selection fusion technique. Inverse transform is applied and then Iterative segmentation is carried out on fused image to get results in the form of relative bias, maximum drop size, minimum drop size, number of raindrops in a given image.
\end{abstract}

Keywords: Raindrop analysis,DWT, image processing,double density dual tree transform, threshold Iterative segmentation.

\section{Introduction}

Every year rain blesses planet earth with life. Rainfall differs from year to year and region to region. In tropical countries like India, diversity has been observed in rainfall. Rain, also known as monsoon in India, impacts elements like soil erosion, weather forecast, telecommunication networks, etc. Previous work proposed for rainfall measurement include traditional techniques such as stain methods, flour pellet method and oil immersion method, whereas modern techniques include acoustic disdrometer and optical disdrometer[1]. Main focus of previous work performed was on disdrometers. This paper performs raindrop size estimation by using $2 \mathrm{D}$ double density dual tree DWT.

Disdrometer is an instrument designed to capture rain in all its forms such as drizzle or heavy rain. One of the techniques under optical disdrometer is optical imaging. Two cameras are aligned in such a way that they capture images at the same time covering same region. While capturing images, the background should be dark so that every small raindrop is visible and distinguishable from the others.

Further part of the paper deals with DWT. Discrete Wavelet Transform (DWT) can be used in various fields such as steganography, biomedical applications and image processing. DWT is a function which samples given signal discretely. DWT for continuous signal $\mathrm{x}(\mathrm{t})$ is given as follow:

$$
\operatorname{DWT}(\mathrm{p}, \mathrm{q})=\int_{-\infty}^{+\infty} \mathrm{x}(\mathrm{t}) \cdot \Psi \mathrm{p}, \mathrm{q} d \mathrm{t}
$$

where $\Psi \mathrm{p}, \mathrm{q}$ is wavelet function

DWT for discrete signal $\mathrm{x}[\mathrm{n}]$ is as follows:

$$
\operatorname{DWT}(\mathrm{p}, \mathrm{q})=\frac{1}{\sqrt{\mathrm{a}}} \sum \mathrm{x}[\mathrm{n}] \cdot \mathrm{g}\left(\frac{\mathrm{q}-\mathrm{nb}_{0 \mathrm{a}_{0}^{\mathrm{p}}}}{\mathrm{a}_{0}^{\mathrm{p}}}\right)
$$

To obtain DWT, signal $x$ ..passes through series of high pass and low pass filters. These filters are known as filter banks.

B. Iyer, S. Nalbalwar and R. Pawade (Eds.)

ICCASP/ICMMD-2016. Advances in Intelligent Systems Research.

Vol. 137, Pp. 412-419.

(C) 2017- The authors. Published by Atlantis Press

This is an open access article under the CC BY-NC license (http://creativecommons.org/licens)es/by-nc/4. 


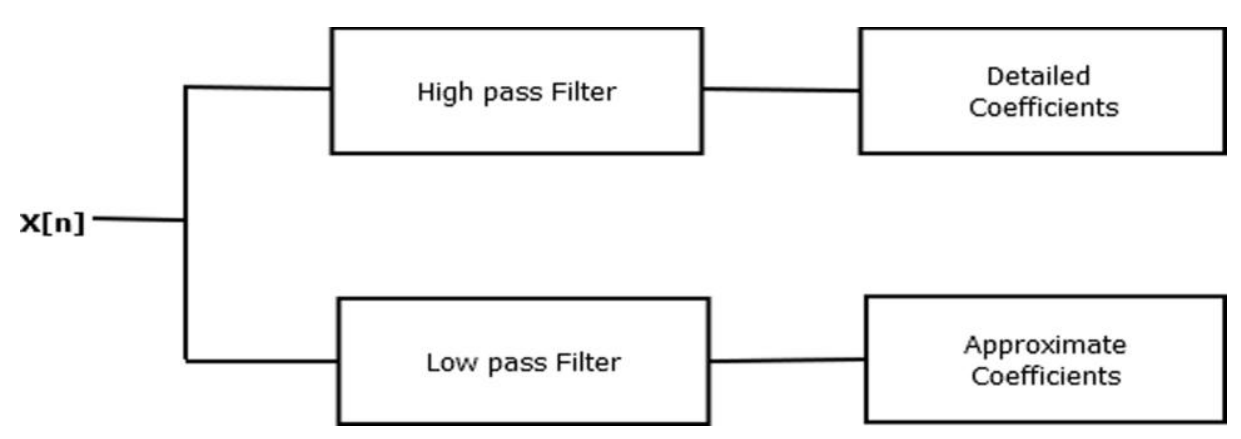

Fig.1. Filter Analysis for Discrete Wavelet Transform

A variant of DWT, which is known as $2 \mathrm{D}$ DWT $\Phi(m, n)$ is a single scaling function.

$$
\Phi(m, n)=\Phi(m) \cdot \Phi(n)
$$

This transform was further enhanced into member of over-complete family of DWT, which is known as 2D double density dual tree DWT. This transform is four times as expansive compared to DWT. Two over-complete transforms such as Dual tree DWT and Double density are combined together to sample the given data.

Equation for dual tree is given as follows:

It is obtained by DWT which outputs Hilbert transform pair

$$
\Psi_{\mathrm{g}}(t) \approx H\left\{\Psi_{\mathrm{h}}(t)\right\}
$$

Equation for double density is given as follows:

This includes a single scaling function $\Phi(\mathrm{t})$ and two wavelets $\Psi_{1}(\mathrm{t})$ and $\Psi_{2}(\mathrm{t})$, where one wavelet is offset from another wavelet by half its value. Integer value of one wavelet is between the integer values of other wavelet.

$$
\begin{array}{r}
\Psi_{2}(t) \approx \Psi_{1}(t-0.5) \\
\Phi(t)=\sqrt{2} \sum_{n} g_{i}(n) \Phi(2 t-n) \\
\Psi_{\mathrm{g}}(\mathrm{t})=\sqrt{2} \sum_{n} h_{i}(n) \Phi(2 t-n), \mathrm{i}=1,2, \ldots
\end{array}
$$

Where, $g(n)$ is low pass filter bank and $h(n)$ high pass filter bank.

\subsection{Literature Survey}

Kai Xie et al. [2] carried out an experiment in China, where rain drop size distribution and velocity of raindrop were measured using parsivel - EF disdrometer. Kai xie et al., not stopping at said result, extended the experiment to obtain raindrop size distribution spectrum. Kai Xie obtained results in the form of values such as ,maximum raindrop diameter and peak value of raindrop size distribution. Kai observed that, as region changes, statistical properties of rain change.

Chen et al. [3] has proposed a technique for tracking and modeling of raindrop to predict rain attenuation. Chen's work is based on improvement of disdrometer technique by applying data processing to it. A new image processing technique is proposed by Chen, which involves capturing image using CCD camera to calculate parameters of rain such as rain rate, rain accumulation, raindrop size distribution and raindrop velocity distribution. Threshold segmentation method used by Chen removes noises present in an image. Instead of assessing single image for analysis, two consecutive frames of same image are assessed. Lam et al. [4] has performed raindrop analysis in Kuala Lumpur, Malaysia using Joss and Waldvogel disdrometer. 
Drop size distribution data was collected and then analyzed to figure out the pattern of rain and maximum attenuation obtained by rain in Malaysia. Lam has performed this experiment to investigate raindrop size distribution in tropical and equatorial regions.

\section{Double Density Dual Tree Discrete Wavelet Transform}

This paper is divided into two sections. First section deals with capturing image through digital imaging technique employed under optical disdrometer. To the best of my knowledge and study, raindrop analysis, till date, is performed by disdrometer. This paper explores new technique of raindrop analysis, output of which resembles disdrometer output. The technique proposed in this paper is based on image processing. To be precise, it is based on Discrete Wavelet Transform (DWT) concept of image processing. Image processing is wide area of work where different algorithms are available, which can be applied for the given problem statement.

The experimental setup to capture images of rain was setup at Regional Meteorological Centre, Mumbai. Two DSLR cameras were fixed with certain angle so that the captured images have same central point. Sample images taken from DSLR were categorized as left focus images and right focus images. As the name suggests, left focus image is obtained from left angled camera and right focus image is obtained from right angled camera, such that both images have same central point.
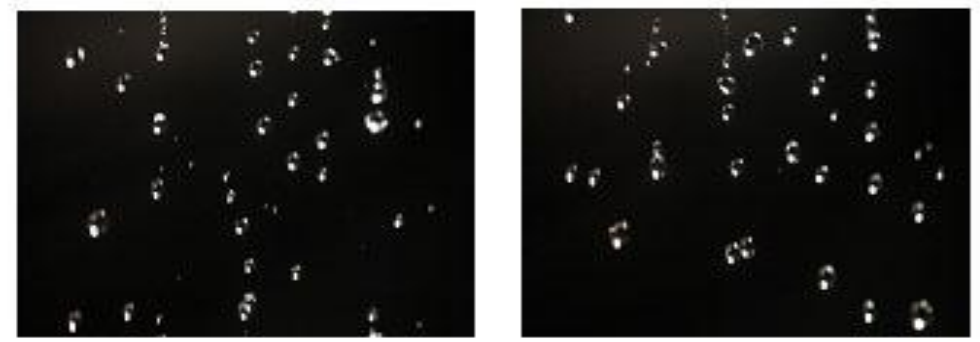

Fig.2. Right Focused Image

Fig.3. Left Focused Image

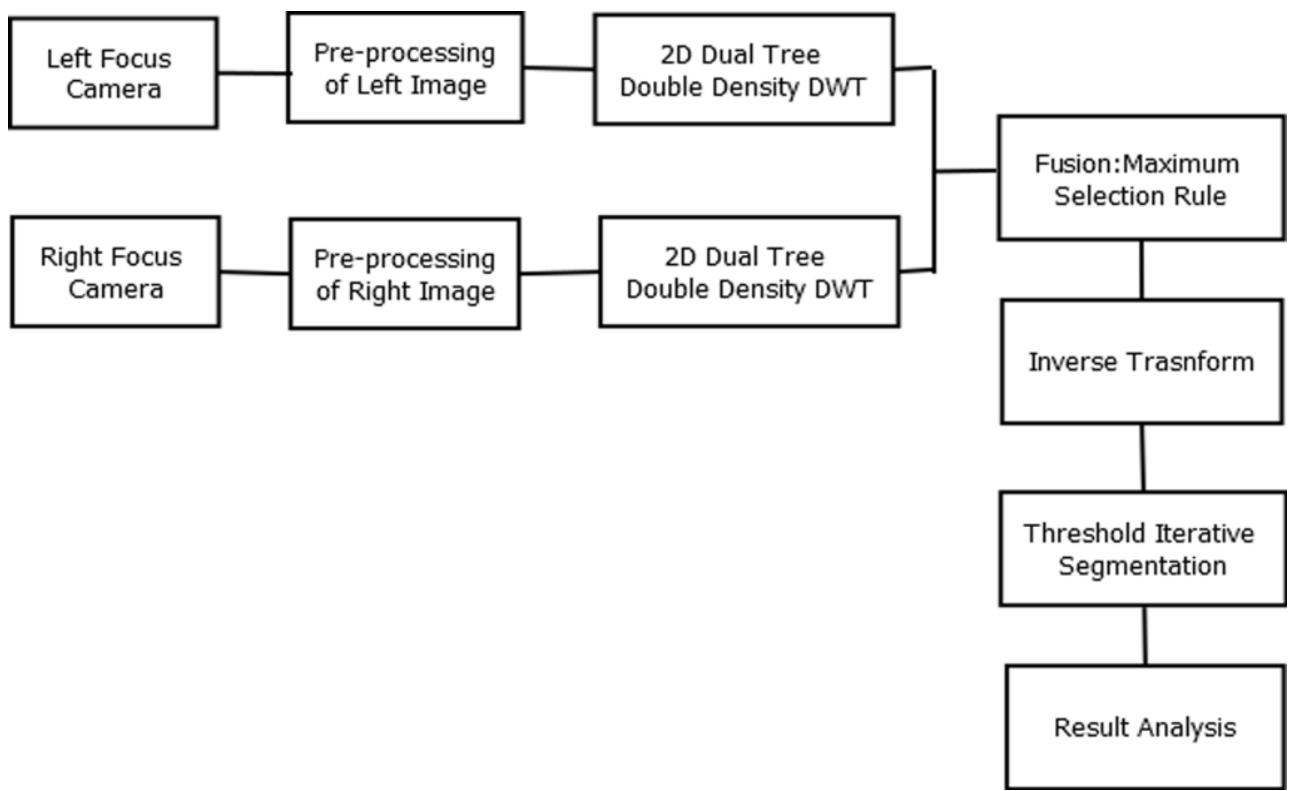

Fig .4. Block Diagram of Raindrop analysis 


\subsection{Pre-processing}

Unprocessed images are first subjected to various stages involved in pre-processing. These steps include the following:

- RGB to gray: Even though for experiment purpose data has been analyzed on small scale for real time application like video screening, a large amount of data is required to be processed. To reduce the amount of processing, multi-chrome images are converted to gray-scale, so that analysis will be performed on $1 / 4$ of the data since $3 / 4$ of the data that includes the chrome component (RGB) is removed.

- Filtering: Filters are used to separate detailed components and approximate components of the wavelet[5]. Median Filter in image processing removes noise from the image. Window of pixels is selected, so that the filter moves from pixel to pixel, sorting them in numerical order. From this window of pixels, the median is selected i.e. pixel from middle.

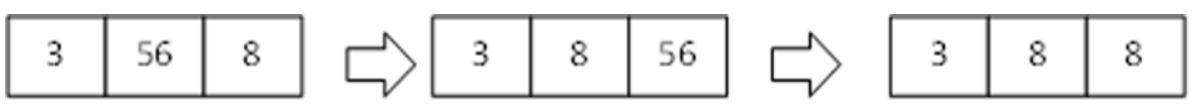

Fig.5. Median Filter

\section{Proposed System}

Dual tree Double density DWT uses iterative filter bank designed by Ivan et al. [6]. Filter bank of two over sampled DWT which run in parallel are combined together such that they run in iteration.

In double density DWT there are two wavelet function s eq.(5) and one scaling function eq.(6)

To design filters, the equations are modified as

$$
\Psi_{\mathrm{h}, \mathrm{i}}(t), \Psi_{\mathrm{g}, \mathrm{i}}(t), \quad i=1,2
$$

Two wavelets functions are offset from each other by half the integer factor

$$
\Psi_{\mathrm{h}, 1}(t) \approx \Psi_{\mathrm{h}, 2}(t-0.5)
$$

$\Psi_{\mathrm{g}, 1}(t)$ is similarly defined. In dual density DWT, two wavelet functions should form Hilbert transform pair from eq.(4)

$$
\begin{aligned}
& \Psi_{\mathrm{g}, 1}(t) \approx H\left\{\Psi_{\mathrm{h}, 1}(t)\right\} \\
& \Psi_{\mathrm{g}, 2}(t) \approx H\left\{\Psi_{\mathrm{h}, 2}(t)\right\}
\end{aligned}
$$

The design of filter constructed by Ivan et al. [6] is based on the following conditions:

1) Filters have perfect reconstruction property.

2) Filters have approximate Hilbert transform pair.

3) Vanishing moment specified for filters should be achieved.

4) Filters made up of short support.

$\mathrm{Z}$ transform for $h_{i}(n)$ and $g_{i}(n)$ are denoted by $H_{i}(z)$ and $G_{i}(z)$

$$
H_{i}(z)=Z T\left\{h_{i}(n)\right\}:=\sum_{n} h_{i}(n) Z^{-n}
$$

where $G_{i}(z)$ is similarly defined. Frequency response is denoted by

$$
\mathrm{H}_{\mathrm{i}}\left(\mathrm{e}^{\mathrm{jw}}\right)=\operatorname{DTFT}\left\{\mathrm{h}_{\mathrm{i}}(\mathrm{n})\right\}:=\sum \mathrm{h}_{\mathrm{i}}(\mathrm{n}) \mathrm{e}^{-\mathrm{jnw}}
$$

where $G_{i}\left(e^{j w}\right)$ is similarly defined. Perfect reconstruction is defined as [10] 
- when filters have finite impulse response $g_{1}(n)$ and $g_{2}(n)$, can reconstruct $h_{1}(n)$ and $h_{2}(n)$

- $\quad$ when output(DWT $)=\operatorname{Input}(D W T)$ and $g_{1}(n)=g_{2}(n), h_{1}(n)=h_{2}(n)$ whereg(n) and $h(n)$ are called as conjugate mirror filters [9].

Perfect Reconstruction conditions for double density dual tree filters given by Ivan et al. [6] are as follows:

$$
\begin{aligned}
& \sum_{i=1}^{2} H_{i}(Z) H_{i}(1 / Z)=2 \\
& \sum_{i=0}^{2} H_{i}(Z) H_{i}(-1 / Z)=0
\end{aligned}
$$

where $G_{i}(Z)$ is similarly defined. Scaling and wavelet functions for filter banks are as follows:

$$
\begin{aligned}
& \Phi_{\mathrm{h}}(\mathrm{t})=\sqrt{2} \sum_{\mathrm{n}} \mathrm{h}_{0}(\mathrm{n}) \Phi_{\mathrm{h}}(2 \mathrm{t}-\mathrm{n}) \\
& \Psi_{\mathrm{h}, 1}(\mathrm{t})=\sqrt{2} \sum_{\mathrm{n}} \mathrm{h}_{1}(\mathrm{n}) \Phi_{\mathrm{h}}(2 \mathrm{t}-\mathrm{n}) \\
& \Psi_{\mathrm{h}, 2}(\mathrm{t})=\sqrt{2} \sum_{\mathrm{n}} \mathrm{h}_{2}(\mathrm{n}) \phi_{\mathrm{h}}(2 \mathrm{t}-\mathrm{n})
\end{aligned}
$$

where $\Phi_{\mathrm{g}}(\mathrm{t}), \Psi_{\mathrm{g}, 1}(\mathrm{t}), \Psi_{\mathrm{g}, 2}(\mathrm{t})$ are similarly defined.By applying Fourier transform for eq.16 to 18 we get following

$$
\begin{aligned}
& \phi_{\mathrm{h}}(\omega)=\mathcal{F}\left\{\phi_{\mathrm{h}}(\mathrm{t})\right\}, \phi_{\mathrm{g}}(\omega)=\mathcal{F}\left\{\phi_{\mathrm{h}}(\mathrm{t})\right\} \\
& \psi_{\mathrm{h}, \mathrm{i}}(\omega)=\mathcal{F}\left\{\psi_{\mathrm{h}, \mathrm{i}}(\mathrm{t})\right\}, \quad \psi_{\mathrm{g}, \mathrm{i}}(\omega)=\mathcal{F}\left\{\psi_{\mathrm{g}, \mathrm{i}}(\mathrm{t})\right\}
\end{aligned}
$$

To build Hilbert transform pair,

$\psi_{g, 1}(t)=H\left\{\psi_{h, 1}(t)\right\}$

$$
\psi_{g, 2}(t)=H\left\{\psi_{h, 2}(t)\right\}
$$

This implies that,

$$
\psi_{\mathrm{g}, \mathrm{i}}(\omega)=\left\{\begin{array}{c}
-\mathrm{j} \psi_{\mathrm{h}, \mathrm{i}}(\omega), \omega>0 \\
j \psi_{\mathrm{h}, \mathrm{i}}(\omega), \omega>0
\end{array}\right\}
$$

Where $\psi_{\mathrm{g}, \mathrm{i}}(\omega)$ is similarly defined. The zero vanishing condition is

$$
\int \mathrm{t}^{\mathrm{k}} \psi_{\mathrm{h}, \mathrm{i}}(\mathrm{t}) \mathrm{dt}=\int \mathrm{t}^{\mathrm{k}} \Psi_{\mathrm{g}, \mathrm{i}}(\mathrm{t}) \mathrm{dt}=0, \quad \mathrm{i}=1,2
$$

Ivan finally proposed following filter banks

$$
\begin{aligned}
& \mathrm{H}_{0}(Z)=\mathrm{D}(Z)\left(1+z^{-1}\right)^{\mathrm{k}_{0}} \mathrm{Q}_{0}(Z) \\
& \mathrm{H}_{1}(Z)=-(Z)^{-\mathrm{L}} \mathrm{D}(-1 / z)\left(1-z^{-1}\right)^{\mathrm{K}_{1}} \mathrm{Q}_{1}(Z) \\
& \mathrm{H}_{2}(Z)=-(Z)^{-\mathrm{L}} \mathrm{D}(-1 / z)\left(1-z^{-1}\right)^{\mathrm{K}_{2}} \mathrm{Q}_{2}(Z)
\end{aligned}
$$

where $G_{\mathrm{i}}(Z)$ is similarly defined with $\mathrm{i}=0,1,2$. As well as

$$
\begin{aligned}
& \mathrm{h}_{0}(\mathrm{n})=\mathrm{d}(\mathrm{n}) * \mathrm{~S}_{0}(\mathrm{n}) \mathrm{q}_{0}(\mathrm{n}) \\
& \mathrm{h}_{1}(\mathrm{n})=(-1)^{\mathrm{n}} \mathrm{d}(\mathrm{L}-\mathrm{n}) * \mathrm{~S}_{1}(\mathrm{n}) * \mathrm{q}_{1}(\mathrm{n}) \\
& \mathrm{h}_{2}(\mathrm{n})=(-1)^{\mathrm{n}} \mathrm{d}(\mathrm{L}-\mathrm{n}) * \mathrm{~S}_{2}(\mathrm{n}) * \mathrm{q}_{2}(\mathrm{n})
\end{aligned}
$$

where $g_{i}(n)$ is similarly defined with $i=0,1,2$ and

$$
\begin{aligned}
& S_{0}(n)=\left(\frac{K_{0}}{n}\right)=\frac{k_{0} !}{\left(k_{0}-n\right) ! n !} \\
& s_{i}(n)=\left(-1^{n}\right)\left(\frac{k_{i}}{n}\right), i=1,2
\end{aligned}
$$

Next step in the process of raindrop analysis is fusing two transformed images together. For this, the technique used is maximum selection fusion rule [9]. From two images to be fused together, same pixel from both images having maximum intensity is selected and retained in the fused image.

Fusion algorithm gives output as a fused image which is then given as an input to the iterative threshold segmentation algorithm. This method is also known as Otsu's method [7]. Otsu's method divides the image to be segmented, into two parts i.e. foreground and background. Then the intra class variances between these two parts are estimated. Otsu's method explores the entire image pixel by pixel till the intra class variance hits the minimum value. This minimum value is considered to be the threshold value for that segmentation process. 


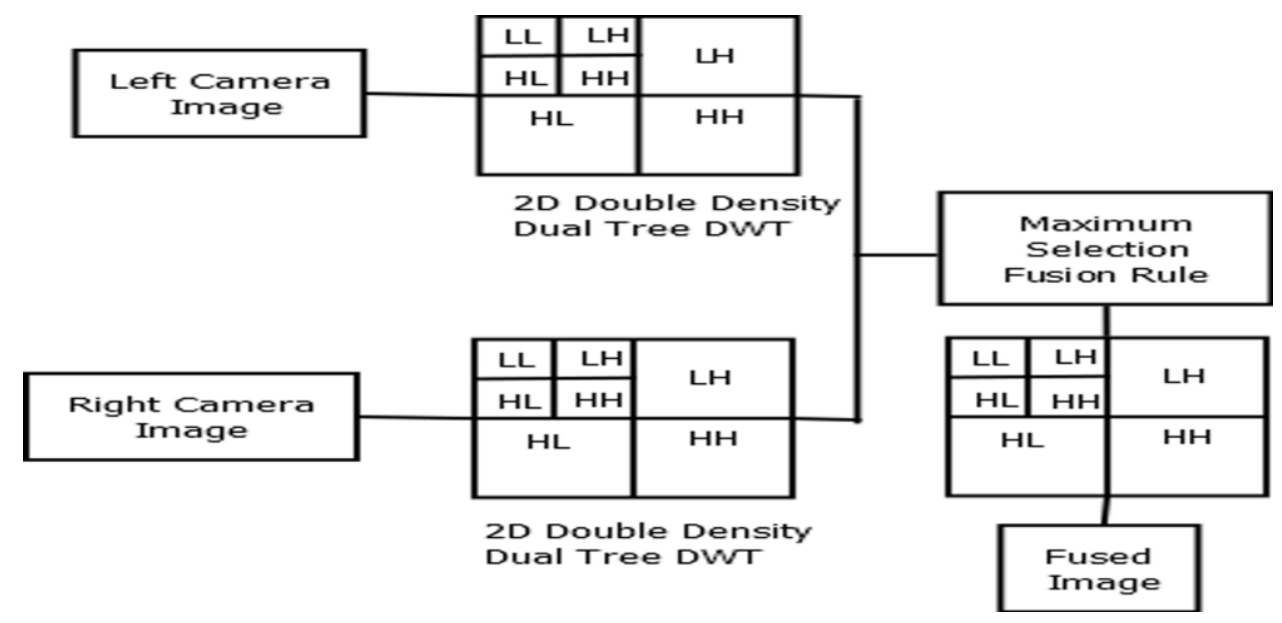

Fig.6. Block Diagram of Fusion Technique

If we consider background as $\mu_{0}$ is darker than foreground $\mu_{1}$ i.e. $\mu_{1}>\mu_{0}$ thenThreshold value is given as follows [7]:

where

$$
\mathrm{T}=\arg \min \sigma_{\mathrm{w}}^{2}(\mathrm{~T})
$$

$$
\begin{aligned}
& \sigma_{\mathrm{w}}^{2}(\mathrm{~T})=\mathrm{q}_{0}(\mathrm{~T}) \sigma_{0}^{2}(\mathrm{~T})+\mathrm{q}_{1}(\mathrm{~T}) \sigma_{1}^{2}(\mathrm{~T}) \\
& \mathrm{q}_{0}=\sum_{\mathrm{i}=1}^{\mathrm{T}} \mathrm{P}(\mathrm{i}), \quad \mathrm{q}_{1}=\sum_{\mathrm{i}=\mathrm{T}}^{\mathrm{K}} \mathrm{P}(\mathrm{i})
\end{aligned}
$$

Where $\mathrm{K}$ is pixel value and Class variance can be given as

$\sigma_{0}^{2}(T)=\sum_{i=1}^{T}\left[i-\mu_{0}(T)\right]^{2} \frac{P(i)}{q_{0}(T)}$

where $\sigma_{1}^{2}(T)$ is similarly defined. Final step is Inverse 2D dual tree double density, which is deployed after segmentation.

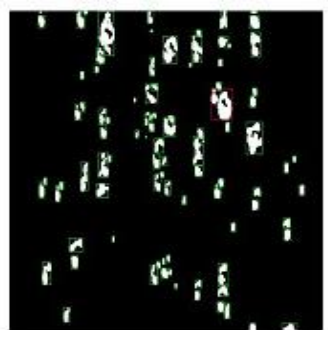

Fig. 7. Fused Image

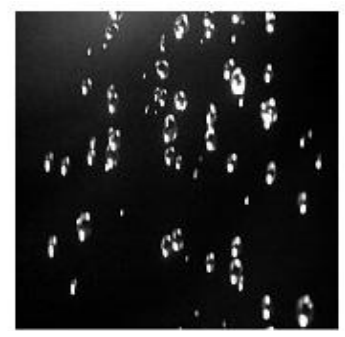

Fig.8. Segmented Image

\section{Discussion and Conclusion}

Most of the existing work performed on raindrop analysis is based on disdrometer. Shape of raindrop, detection of raindrop, analysis of rainfall on data obtained from disdrometer are some of the points that the previous work have already contributed in raindrop analysis domain. In this paper, a new method which inherits properties of disdrometer for raindrop measurement is proposed. This paper gives step by step design of raindrop analysis method.Propsed method uses transform technique in addition to fusion, segmentation to extract following properties from fused images. 
Table 1. Raindrop Parameters

\begin{tabular}{clcc}
\hline Sr. No & \multicolumn{1}{c}{ Parameters } & $\begin{array}{c}\text { Using } \\
\text { DDDTDWT }\end{array}$ & $\begin{array}{c}\text { Without using } \\
\text { DDDTDWT }\end{array}$ \\
\hline 1 & Maximum drop size in mm2 & 6.741053 & 5.345593 \\
2 & Minimum drop size in mm2 & 0.264000 & 0.264000 \\
3 & Volume of maximum drop size & 4.599053 & 5.744107 \\
4 & Volume of raindrop in fused image & 35.86717 & 30.94574 \\
5 & Total no of rain drop & 114 & 141
\end{tabular}

\section{Abbreviation}

$\begin{array}{lll}\text { 1. } & \text { DWT- } & \text { Discrete Wavelet Transform } \\ \text { 2. } & \text { DDDTDWT - } & \text { Double density Dual tree DWT } \\ \text { 3. } & \text { DSLR- } & \text { Digital Single-Lens Reflex Camera } \\ \text { 4. } & \text { RGB- } & \text { Red-Green-Blue } \\ \text { 5. } & \text { ZT- } & \text { Z Transform } \\ \text { 6. } & \text { DTFT- } & \text { Discrete-Time Fourier Transform } \\ \text { 7. } & \text { LL- } & \text { Low-Low } \\ \text { 8. } & \text { LH- } & \text { Low High } \\ \text { 9. } & \text { HL- } & \text { High Low } \\ \text { 10. } & \text { HH- } & \text { High High }\end{array}$

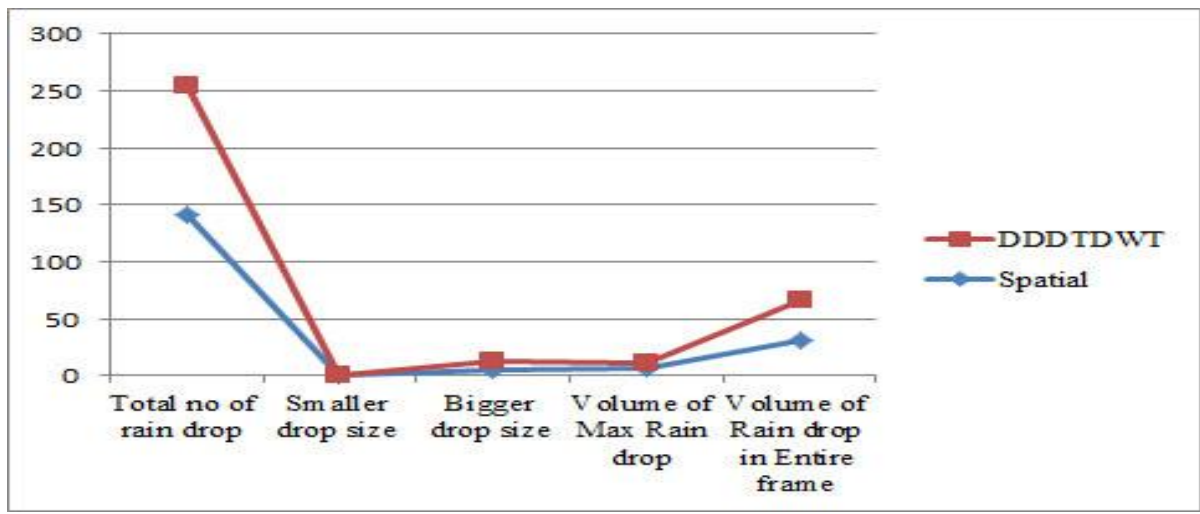

Fig.9. Raindrop Parameters-DDDTDWT V/S Spatial Transform

Result produced using DDDTDWT shows great improvement over techniques where raindrop analysis is performed in spatial domain i.e. without using any transform techniques.

Table 2. Fusion Parameters

\begin{tabular}{llll}
\hline Sr. No & Parameters & Using DDDTDWT & $\begin{array}{l}\text { Without using } \\
\text { DDDTDWT }\end{array}$ \\
\hline 1 & Relative Bias & 0.3013 & 0.26 \\
2 & Relative Variance & 0.6637 & 0.7585 \\
3 & Standard Deviation & 6.5597 & 5.782 \\
4 & Root Mean Square Error & 0.7203 & 0.4567 \\
5 & Percentage Residual Difference & 0.0186 & 0.0153 \\
6 & Peak Signal to Noise Ratio & 49.5556 & 51.5344 \\
7 & Correlation Coefficient & 0.7653 & 0.7497 \\
8 & Quality Index & 12.3659 & 11.8492
\end{tabular}




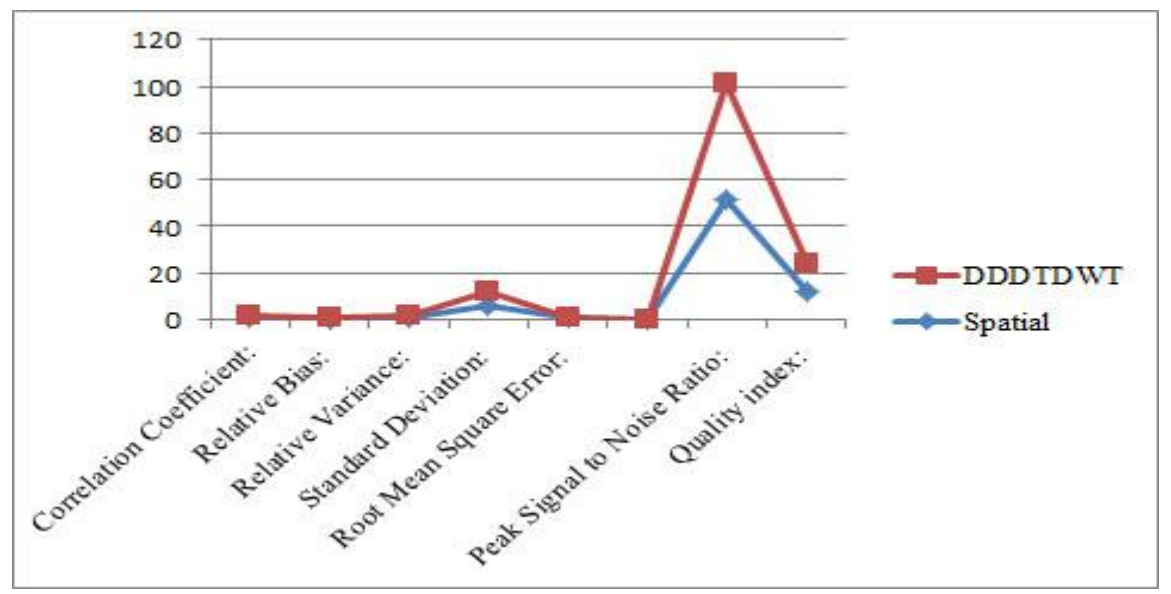

Fig.10. Fusion Parameters-DDDTDWT V/S Spatial Transform

As shown in graph DDDTDWT gives large improvement over spatial domain implementation of raindrop measurement technique in both raindrop parameters and fusion parameters.

\section{References}

[1] Gopinath Kathiravelu, Terry Lucke , and Peter Nichols Rain Drop Measurement Techniques: A Review MDPI .journal ,2016

[2] Kai Xie, Shuhong Gong, Xiaoping Li Lina Zhang, Measurement and Analysis of Raindrop Size Distribution in Xi'an, IEEE 2010

[3] Chih-Yen Chen, Chun-Jen Weng, Chi-Hung Hwang Tracking and modeling raindrops in video sequences for assessing precipitation, IEEE 20116

[4] H.Y. Lam, Din.J, L Luini, A. D. Panagopoulos , C. Capsoni Analysis of Raindrop Size Distribution Characteristics in Malaysia for Rain Attenuation Prediction, IEEE 2011

[5] Satishkumar S. Chavan, Sanjay N. Talbar, Multimodality Image Fusion in Frequency Domain for Radiation Therapy, International Conference on Medical Imaging, m-Health and Emerging Communication Systems ,2014

[6] Ivan W. Selesnick, The Double-Density Dual-Tree DWT, IEEE Transactions on Signal Processing, Vol. 52, No. 5, May 2004

[7] Hongmin Cai, Zhong Yang, Xinhua Cao A New Iterative Triclass Thresholding Technique in Image Segmentation, IEEE Transactions On Image Processing, Vol. 23, No. 3, March 2014

[8] Bogdan Dumitrescu, Ali Bahrami Rad, A METHOD FOR DESIGNING THE DOUBLE-DENSITY DUAL-TREE DISCRETE WAVELET TRANSFORM BIORTHOGONAL WAVELET

[9] Om Prakash1,2, Richa Srivastava1, Ashish Khare1, TRANSFORM BASED IMAGE FUSION USING ABSOLUTE MAXIMUM FUSION RULE, Proceedings of 2013 IEEE Conference on Information and Communication Technologies

[10] http://cas.ensmp.fr/ chaplais/wavetour_presentation/filtres/Perfect_Reconstruction.html 